\title{
PSEUDO SU(3) APPROXIMATION FOR $\alpha$-SPECTROSCOPIC FACTORS
}

\author{
D. BRAUNSCHWEIG and K.T. HECHT ${ }^{1}$ \\ Physics Department, University of Michigan, Ann Arbor, MI 48109, USA \\ and \\ J.P. DRAAYER ${ }^{1}$ \\ Department of Physics and Astronomy, Louisiana State University, Baton Rouge, LA 70803, USA
}

Received 6 March 1978

Revised manuscript received 4 May 1978

\begin{abstract}
Estimates for $\alpha$-transfer strengths based on the pseudo SU(3) model are tested. $\alpha$-cluster functions are shown to have large overlaps with pseudo SU(3) representations of highest symmetry. Pseudo SU(3) estimates are compared with $\mathrm{f}_{5 / 2} \mathrm{p}_{3 / 2} \mathrm{p}_{1 / 2}$ shell model predictions for $\alpha$-transfers from Ni to $\mathrm{Zn}$ isotopes.
\end{abstract}

Many data have recently been accumulated on $\alpha$ particle spectroscopic strengths in both sd shell [1-3] and $\mathrm{fp}$ shell [4] nuclei. Since the wave function for the $\alpha-(A-4)$ relative motion in an $A$-particle nucleus carries a single SU(3) representation [5], and since the $\alpha$ spectroscopic strength is highly concentrated in a few rotational bands in nuclei of good SU(3) symmetry, analyses in terms of the SU(3) shell model $[5,6]$ have been very fruitful for nuclei in the first half of the sd shell. Predictions based on the simplest SU(3) approximations, in which both initial and final nuclear wave functions are assumed to have pure SU(3)-SU(4) symmetry, have been remarkably successful in explaining both the ground state systematics [7] and the relative strengths within the lowest rotational bands [1] and are moreover in good agreement with much more detailed shell model calculations [8]. SU(3) symmetry breaks down in heavier nuclei. Consequently it is more difficult to exploit the inherent SU(3) symmetry of the $\alpha$-transfer process valid for reaction analyses based on the assumption of a single-cluster transfer mechanism with relative motion function carrying a definite number of oscillator quanta (fixed $2 N+L$ ).

It is the purpose of this note to investigate the pos-

\footnotetext{
${ }^{1}$ Supported by the National Science Foundation.
}

sibility that the pseudo SU(3) model $[9,10]$ may furnish a simple means for semiquantitative estimates of $\alpha$-transfer strengths in heavier nuclei. For this purpose recent experimental results [4] and analyses [11] for nuclei in the $\mathrm{f}_{5 / 2} \mathrm{p}_{3 / 2} \mathrm{p}_{1 / 2}$ shell with $A>56$ are of special interest, particularly since the $\alpha$-transfers from ground states to low-lying final states account for relatively high percentages of sum rule limits, indicating a shell model structure with considerable $\alpha$-clustering. In addition, Akiyama et al. [12] have shown that nuclei in the $\mathrm{Cu}$ and $\mathrm{Zn}$ region of the $\mathrm{f}_{5 / 2} \mathrm{p}_{3 / 2} \mathrm{p}_{1 / 2}$ or pseudo sd shell have low lying states of good pseudo $\mathrm{SU}(3)$ symmetry rivalling that of the corresponding $\mathrm{F}$ and $\mathrm{Ne}$ isotopes in the real sd shell. In making the association between the real $\mathrm{f}_{5 / 2} \mathrm{p}_{3 / 2} \mathrm{p}_{1 / 2}$ and pseudo $\tilde{d}_{5 / 2} \widetilde{d}_{3 / 2} \tilde{s}_{1 / 2}$ wave functions it is important to fix the relative phases of the single particle wave functions such as to optimize the goodness of the pseudo coupling. Of the 4 possible choices for these relative phases by far the best pseudo SU(3) symmetry results when the $f_{5 / 2}$ and $\widetilde{d}_{5 / 2}$, and $p_{1 / 2}$ and $\widetilde{s}_{1 / 2}$ wave functions have similar magnitudes and signs in the surface region of the nucleus [12]. (Earlier estimates of the pseudo $S U(3)$ content of ${ }^{59} \mathrm{Cu}$ and ${ }^{60} \mathrm{Zn}$ by Strottman [13], have been recalculated with revised phases [14], and are now in good agreement with 
Table 1

Overlaps of the $(\mathrm{fp})^{4}(\lambda \mu)=(12,0) \alpha$-cluster function with the $(\widetilde{s d})^{4}(\widetilde{\lambda \mu})=(80)$ 4-particle functions.

\begin{tabular}{lll}
\hline$L$ & $\langle(\widetilde{80}) L \mid(12,0) L\rangle$ & $\langle(\widetilde{80}) L \mid(12,0) L\rangle \mathrm{f}_{5 / 2} \mathrm{p}_{3 / 2} \mathrm{p}_{1 / 2}$ a) \\
\hline 0 & 0.61 & 0.83 \\
2 & 0.58 & 0.85 \\
4 & 0.52 & 0.87 \\
6 & 0.42 & 0.91 \\
8 & 0.27 & 0.96 \\
\hline
\end{tabular}

a) Column 2 lists the overlap of $\langle(\widetilde{80}) L|$ with a function obtained by renormalizing to unity the $\left(f_{5 / 2} p_{3 / 2} p_{1 / 2}\right)^{4}$ part of $|(12,0) L\rangle$.

those of Akiyama et al. [12]. Approximately 70\% of the ground state of ${ }^{60} \mathrm{Zn}$, e.g., is made up of the leading pseudo $S U(3)$ representation (80), pseudo space symmetry [4].) To show that the pseudo SU(3) scheme can lead to simple estimates of $\alpha$-transfer strengths, in complete analogy with $\mathrm{SU}(3)$ in the real sd shell, it is necessary, in addition, to show that the 4-particle $\alpha$-cluster functions are dominated by a single pseudo SU(3) representation of highest possible symmetry. One purpose of this note is to show that this requirement is satisfied. Paradoxically, however, we also show that, although the simplest pseudo SU(3) approximation yields relative strengths in excellent agreement with predictions based on detailed $\mathrm{f}_{5 / 2} \mathrm{p}_{3 / 2} \mathrm{p}_{1 / 2}$ shell model calculations, differences in absolute strengths are large. These differences signal the importance of lower pseudo symmetry admix tures in the nuclear wave function and in the $\alpha$-transfer operator.

The 4-nucleon wave function for an $\alpha$-cluster transferred into a real fp-shell configuration has pure SU(3) symmetry, $(\lambda \mu)=(12,0)$, provided the $\alpha$-cluster has the same size in both projectile and residual nuclei. When projected onto the $f_{5 / 2} p_{3 / 2} p_{1 / 2}$ or pseudo $\widetilde{s d}$ shell subspace, this function is a linear combination of all possible 4-nucleon pseudo SU(3)-SU(4) representations with $T=0$. Table 1 shows, however, that it is dominated by the leading representation $(\tilde{\lambda \mu})=(80)$, $[\tilde{\mathrm{f}}]=[4] \widetilde{S}=T=0$. Column 1 of table 1 gives the overlap of the normalized $(\widetilde{\mathrm{sd}})^{4}(\widetilde{\lambda \mu})=(80)$ wave function with the full $(\mathrm{fp})^{4}(\lambda \mu)=(12,0) \alpha$-cluster wave function. Column 2 gives the overlap of $(\widetilde{\mathrm{sd}})^{4}(\tilde{\lambda \mu})$ $=(80)$ with a renormalized $(\mathrm{fp})^{4}(\lambda \mu)=(12,0)$ function in which all components $\left(\mathrm{f}_{7 / 2}\right)^{n}\left(\mathrm{f}_{5 / 2} \mathrm{p}_{3 / 2} \mathrm{p}_{1 / 2}\right)^{4-n}$ with $n \neq 0$ have been set equal to zero. Since only components with $n=0$ can contribute to the $\alpha$-spectroscopic strength for $\alpha$-transfer into a nucleus with a full $f_{7 / 2}$ shell only the pseudo SU(3) content of this part of the $(\mathrm{fp})^{4}(\lambda \mu)=(12,0)$ function is of relevance for $\alpha$-transfer into such a nucleus. The results of table 1 show that the $(\tilde{\lambda \mu})=(80) \widetilde{\mathrm{sd}^{4}}$ wave function is not only the major component of this part of the $(f p)^{4}(\lambda \mu)$ $=(12,0)$ function, but that these $(\widetilde{80})$ overlaps are only mildly dependent on $L$. Moreover, these $(\widetilde{80})$ overlaps which vary from 0.83 for $L=0$ to 0.96 for $L$ $=8$, are very similar to the $(\widetilde{80})$ overlaps of the eigenfunctions of the $(\widetilde{\mathrm{sd}})^{4}$ nucleus ${ }^{60} \mathrm{Zn}$ resulting from the diagonalization of a realistic effective interaction hamiltonian [12]. The overlap between the $(\widetilde{\mathrm{sd}})^{4}(\tilde{\lambda \mu})$ $=(80)$ wave function and the renormalized $\mathrm{f}_{5 / 2} \mathrm{p}_{3 / 2} \mathrm{p}_{1 / 2}$ part of the real $(\lambda \mu)=(12,0)$ function is 0.83 for the $J=0$ state when $(\widetilde{\lambda \mu})=(80)$ is defined, as it is in table 1 , using the physically meaningful phase choices discussed above. This overlap of 0.83 would be reduced to $0.46,0.35$, or 0.33 for the $J=0$ states of the three other possible pseudo SU(3) symmetries, where these are obtained by changing the relative phases of both $f$ to $p$ and $\widetilde{s}$ to $\widetilde{d}$, or only $f$ to $p$, or only $\widetilde{s}$ to $\widetilde{d}$ (in that order for the three numbers listed). This again points out the importance of the proper choice of phases in making the association between the real $\mathrm{f}_{5 / 2} \mathrm{p}_{3 / 2} \mathrm{p}_{1 / 2}$ and pseudo $\widetilde{\mathrm{d}}_{5 / 2} \widetilde{\mathrm{d}}_{3 / 2} \widetilde{\mathrm{s}}_{1 / 2}$ functions. With the definition of $(\widetilde{\lambda \mu})=(80)$ which is physically meaningful [i.e. with phase choices which make $(\widetilde{80})$ dominant in the $(\lambda \mu)=(12,0)$ wave function] , the remainder of the $(12,0)$ intensity is concentrated partly in the next highest $(\tilde{\lambda \mu})$ but has many small components distributed among the remaining pseudo $\mathrm{SU}(3)-\mathrm{SU}(4)$ representations with $T=0$. For the $J=0$ state, e.g., the intensities are $69.6 \%(\tilde{\lambda \mu})=(80)$, pseudo space symmetry [ $\widetilde{4}] \widetilde{L}=\widetilde{S}=0 ; 17.3 \%(\widetilde{\lambda \mu})=(61)[\widetilde{3} 1] \widetilde{L}=\widetilde{S}=1$; $1.7 \%(\widetilde{\lambda \mu})=(42)[\widetilde{4}] \widetilde{L}=\widetilde{S}=0 ; 1.2 \%(\widetilde{\lambda \mu})=(42)[\widetilde{22}]$ $\widetilde{L}=\widetilde{S}=0$; and $0.9 \%(\tilde{\lambda \mu})=(42)[\widetilde{22}] \widetilde{L}=\widetilde{S}=2$; the remainder being fragmented into even smaller pieces among the remaining $(\widetilde{\lambda \mu})[\widetilde{f}] \widetilde{L}, \widetilde{S}$ components.

Since the $(\tilde{\lambda \mu})=(80)$ components have large 4-particle fractional parentage coefficients, particularly between initial and final states of high $(\widetilde{\lambda \mu})$ symmetry, a simple pseudo SU(3) approximation, one in which the small $(\widetilde{\lambda \mu})$ components of the 4-particle transfer operator are neglected al together, may give a reasonable estimate of the $\alpha$-spectroscopic amplitudes for stripping into the $f_{5 / 2} p_{3 / 2} p_{1 / 2}$ configuration of a nucleus with 
Table 2

Comparison of shell model $\alpha$-spectroscopic factors with the pseudo SU(3) approximation. Numbers in columns 1 and 2 give $\alpha$-strengths relative to the ground state transitions. Numbers in parentheses give the absolute ground state predictions.

\begin{tabular}{|c|c|c|c|}
\hline$K$ & $L$ & $\begin{array}{l}S_{\alpha}(\text { pseudo } S U(3) \\
\left.\text { approximation) }^{\text {a }}\right)\end{array}$ & $\begin{array}{l}S_{\alpha}\left(\mathrm{f}_{5 / 2} \mathrm{p}_{3 / 2} \mathrm{p}_{1 / 2}\right. \\
\text { shell model })\end{array}$ \\
\hline
\end{tabular}

${ }^{58} \mathrm{Ni}\left(0^{+}\right) \rightarrow{ }^{62} \mathrm{Zn}\left(\mathrm{L}^{+}\right)[(40) \rightarrow(82)]$

\begin{tabular}{lrll}
0 & 0 & $1.00(0.010)$ & $1.00(0.020)$ \\
0 & 2 & 0.70 & 0.70 \\
0 & 4 & 0.26 & 0.29 \\
2 & 2 & 0.004 & 0.001 \\
2 & 4 & 0.046 & 0.029 \\
60 & & \\
\multicolumn{1}{l}{$\mathrm{Ni}\left(0^{+}\right) \rightarrow{ }^{64} \mathrm{Zn}\left(\mathrm{L}^{+}\right)[(42) \rightarrow(10,0)]$} & \\
0 & 0 & $1.00(0.0039)$ & $1.00(0.018)$ \\
0 & 2 & 0.59 & 0.59 \\
0 & 4 & 0.11 & 0.18 \\
\hline
\end{tabular}

a) The pseudo $\mathrm{SU}(3)$ approximation is given by eq. (1). The assumed initial and final state $(\tilde{\lambda} \tilde{\mu})$ are indicated in [ ] .

b) The shell model predictions are those of Bennett, Fulbright, van Hienen, Chung and Wildenthal [11].

$A>56$, assuming full $\mathrm{f}_{7 / 2}$ shell. With the additional assumption that both initial and final states have pure pseudo SU(3)-SU(4) symmetry, the $\alpha$-spectroscopic factor in this extreme pseudo SU(3) approximation can be estimated from the approximation formula

$$
\begin{aligned}
S_{\alpha} & \approx\left(\frac{A}{A-4}\right)^{8}\langle[4](12,0) L \mid[\widetilde{4}](\widetilde{80}) L\rangle^{2} G^{2}\left((\widetilde{\mathrm{sd}})^{4}\right) \\
& \times A_{\widetilde{L} \widetilde{S} J}^{2}\left\langle[\tilde{\mathrm{f}}]\left(\widetilde{\lambda_{\mathrm{f}} \mu_{\mathrm{f}}}\right)|||| \chi^{+}[\tilde{4}](\widetilde{80})||||[\tilde{\mathrm{i}}]\left(\widetilde{\left.\lambda_{\mathrm{i}} \mu_{\mathrm{i}}\right)}\right\rangle^{2},\right.
\end{aligned}
$$

where the 4-particle cfp's are given in terms of the guadruple-barred reduced matrix elements of $\chi^{+[\widetilde{4}](\widetilde{80})}$ which can be read from the tables of ref. [6] or obtained from ref. [15]. The $A \widetilde{L} \widetilde{S} J$ factors give the dependence on SU(3), SU(4) subgroup labels [6]. For an initial state with $\widetilde{L}=\widetilde{S}=0$ (even nucleus), the

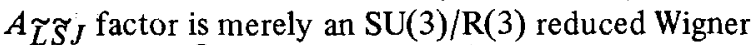
coefficient $\left\langle\left(\widetilde{\lambda_{\mathrm{i}} \mu_{\mathrm{i}}}\right) 0 ;(80) L \|\left(\widetilde{\lambda_{\mathrm{f}} \mu_{\mathrm{f}}}\right) L\right\rangle$. The $G$-factor appearing in the pseudo-sd-shell formula (1) is rigorously identical with that for the real sd shell, $G=[8 ! /$ $\left.(2 !)^{4} 4^{8}\right]^{1 / 2}$. The overlaps between the $(12,0) \alpha$-cluster function and the (80) pseudo SU(3) components can be read from the first column of table 1 . The $[A /$ $(A-4)]^{8}$ factor involves an approximation that is ad- ditional to the assumptions of pure pseudo SU(3)SU(4) symmetry for the initial nuclear state, transferred $\alpha$-state, and final nuclear state. In the real sd shell the factor $[(A-4) / A]^{Q / 2}$ arises from the projection of the true $\alpha$-core relative motion function from a "cluster-like" shell model function [5] which contains spurious center of mass excitations. In the pseudo $\mathrm{SU}(3)$ scheme the corresponding factor now involves a projection in the pseudo orbital space. In contrast with the real sd shell where the projection process transforms precisely from cluster-like to true $\alpha$-core relative motion functions and thus eliminates spurious content, the $[A /(A-4)]^{8}$ factor may now include effects of small components of spurious center of mass excitations in the real orbital space. It is estimated that this leads to errors of no more than 10-20\%.

Complete shell model calculations for $\alpha$-transfer into the $\mathrm{f}_{5 / 2} \mathrm{p}_{3 / 2} \mathrm{p}_{1 / 2}$ shell have recently been carried out by Bennett et al. [11] for nuclei near $A=56$. Table 2 gives a comparison of these shell model predictions with the simple pseudo $\mathrm{SU}(3)$ estimate of eq. (1). In view of the rather extreme nature of the approximations inherent in eq. (1) the agreement, at least for relative strengths, is quite remarkable. The absolute ground state to ground state $\alpha$-strength predictions (numbers in parentheses in table 2), however, give a different impression suggesting the presence of coherent effects missed in the simple pseudo SU(3) picture. To test this an expanded SU(3) calculation was carried out for the ${ }^{58} \mathrm{Ni}($ g.s. $)+\alpha \rightarrow{ }^{62} \mathrm{Zn}$ (g.s.) transfer. In addition to the $[\tilde{f}](\widetilde{\lambda \mu})=[4](80) \widetilde{L}=\widetilde{S}=0(69.6 \%)$ part of the $\alpha$ transfer operator, the $[\widetilde{31}](\widetilde{61}) \widetilde{L}=\widetilde{S}=1(17.3 \%)$ component was taken into account. For $58 \mathrm{Ni}$ (g.s.) the complete $\widetilde{s d}$ wave function was used. For ${ }^{62} \mathrm{Zn}$ the basis included all $(\tilde{\lambda \mu})$ with $2 \widetilde{\lambda}+\tilde{\mu} \geqslant 12$ in the [f] $=[42]$ symmetry and states with $2 \widetilde{\lambda}+\widetilde{\mu} \geqslant 15$ in all other [ $\tilde{\mathrm{f}}]$ (a basis of dimensionality 12 compared to 148 for the full $J=0 \mathrm{f}_{5 / 2} \mathrm{p}_{3 / 2} \mathrm{p}_{1 / 2}$ space). This truncation was chosen since states of large intrinsic deformation are favored by effective interactions strongly correlated with $-\widetilde{Q} \cdot \widetilde{Q}$ such as the ASDI of ref. [11]. The resultant pseudo $\mathrm{SU}(3)$ purity of ${ }^{58} \mathrm{Ni}$ and ${ }^{62} \mathrm{Zn}$ is similar to the SU(3) purity of the analogous real sd shell nuclei ${ }^{18} \mathrm{O}$ and ${ }^{22} \mathrm{Ne}$. Despite the extended basis only a $9 \%$ increase in the predicted $0^{+} \rightarrow 0^{+} \alpha$-strength was realized. The remaining difference in the absolute $\alpha$ strength predictions, a factor of $1 / 1.83$ for ${ }^{58} \mathrm{Ni}+\alpha$ $\rightarrow{ }^{62} \mathrm{Zn}$, is therefore somewhat of a mystery. The dif- 
Table 3

Overlap of the $\left[(\mathrm{sdg})^{2}(80)(\mathrm{pfh})^{2}(10,0)\right](18,0) \alpha$-cluster function with the $\left[(\widetilde{\mathrm{pf}})^{2}(60)(\widetilde{\mathrm{sdg}})^{2}(80)\right](14,0) 4$-particle functions.

\begin{tabular}{lll}
\hline$L$ & $\langle(\widehat{14,0} L|(18,0) L\rangle$ & $\langle(\widehat{14,0}) L \mid(18,0) L\rangle)$ \\
\hline 0 & 0.75 & 0.83 \\
2 & 0.74 & 0.83 \\
4 & 0.72 & 0.84 \\
\hline
\end{tabular}

a) Column 2 lists the overlap of $\langle(\widetilde{14,0}) L|$ with a function obtained by renormalizing to unity that part of $\{(18,0) L\rangle$ having proton part $\left(g_{7 / 2} d_{5 / 2} d_{3 / 2} s_{1 / 2}\right)^{2}$ and neutron part $\left(\mathrm{h}_{9 / 2} \mathrm{f}_{7 / 2} \mathrm{f}_{5 / 2} \mathrm{p}_{3 / 2} \mathrm{p}_{1 / 2}\right)^{2}$.

ference for ${ }^{60} \mathrm{Ni}+\alpha \rightarrow{ }^{64} \mathrm{Zn}$, by the larger factor of $1 / 4.6$, is undoubtedly related partly to lower pseudo SU(3) purity in these more neutron-rich nuclei. The calculations for both $\mathrm{Ni}$ isotopes show that in the $\alpha$ transfers from $\mathrm{Ni}$ to $\mathrm{Zn}$, within an $\mathrm{f}_{5 / 2} \mathrm{p}_{3 / 2} \mathrm{p}_{1 / 2}$ model, contributions from many seemingly small but important components other than the leading $(\widetilde{\lambda \mu})$ in $\mathrm{Ni}$ and $\mathrm{Zn}$, and other than the dominant ( $\widetilde{80}$ ) of the $\alpha$-transfer operator, can combine coherently to yield an $\alpha$-transfer strength considerably greater than that predicted by the zeroth order pseudo-SU(3) approximation. Despite the excellent agreement for relative strengths, the failure of the simple pseudo $\mathrm{SU}(3)$ approximation to reproduce the full shell model predictions for absolute strengths negates the hope of simple easily-generated approximations for $\alpha$-strengths in the $A>56$ region.

Deformed nuclei in the rare earth region may furnish a further testing ground for the simple pseudo $\mathrm{SU}(3)$ approximation for the $\alpha$-transfer process. For rare earth nuclei we have calculated $\alpha$-transfer by using a 4-nucleon wave function with $(\lambda \mu)=(18,0)$, built from an $(\mathrm{sdg})^{2}$ proton (80) pair coupled with an (pfh) ${ }^{2}$ neutron $(10,0)$ pair. (Experimental $\alpha$-spectroscopic factors extracted from recent $\left(\mathrm{d},{ }^{6} \mathrm{Li}\right)$ reaction analyses by Milder et al. [16] have been based on a single-cluster transfer mechanism with $2 N+L=18$ for the transferred $\alpha$-cluster in rare earth nuclei.) Under the assumption that the $g_{9 / 2}$ proton and $h_{11 / 2}$ neutron shells are filled in nuclei in the $A=160$ region, we approximated the $\alpha$-cluster function by a 4 -nucleon function of pseudo SU(3) symmetry $(\widetilde{\lambda \mu})=(14,0)$, built from a $(\widetilde{p f})^{2}$ proton $(60)$ pair coupled with an $(\widetilde{\mathrm{sdg}})^{2}$ neutron (80) pair. Table 3 shows that the overlaps between the $(14,0)$ pseudo $S U(3)$ representation and the $\left(g_{7 / 2} d_{5 / 2} d_{3 / 2} s_{1 / 2}\right)^{2}\left(h_{9 / 2} f_{7 / 2} f_{5 / 2} p_{3 / 2} p_{1 / 2}\right)^{2}$ part of the 4-nucleon $(18,0)$ function are close to unity. Additional assumptions must be made for the symmetries of the initial and final state wave functions. In the strong coupling approximation for the pseudo $\mathrm{SU}(3)$ symmetry, in which neutron and proton $(\widetilde{\lambda \mu})$ 's are coupled to resultant total $(\widetilde{\lambda \mu})$, the extreme pseudo SU(3) approximation predicts $\alpha$-spectroscopic factors which are too small by factors of $\sim 3$ compared with the values of $S_{\alpha}$ extracted from the experimentally observed $\left(\mathrm{d},{ }^{6} \mathrm{Li}\right)$ cross sections [16] for ${ }^{154} \mathrm{Sm}$ and ${ }^{166} \mathrm{Er}$. An SU(3) weak coupling approximation gives still smaller values. Since conventional shell model predictions for $\alpha$-spectroscopic amplitudes, based on pure valence shell model configurations, are known to be smaller than the experimentally observed values in heavy nuclei, the simple pseudo $S U(3)$ approximation for the $\alpha$-spectroscopic strength may nevertheless be a useful estimate for the contributions of the valence shell nucleons. Since the overlap factors of tables 3 and 2 are similar, deviations of the $\alpha$-transfer operator from pure $(\widetilde{\lambda \mu})$ symmetry may lead to difficulties as in the $\widetilde{\text { sd }}$ shell, and the usefulness of the simple pseudo SU(3) approximation remains to be tested further.

\section{References}

[1] N. Anantaraman, H.E. Gove, J. Toke and J.P. Draayer, Nucl. Phys. A279 (1977) 474; Phys. Lett. 60B (1976) 149 ;

N. Anantaraman, H.E. Gove, J.P. Trentelman, J.P. Draayer and F.C. Jundt, Nucl. Phys. A276 (1977) 119 ;

J.P. Draayer, H.E. Gove, J.P. Trentelman, N. Anantaraman and R.M. DeVries, Phys. Lett. 53B (1974) 250.

[2] A.M. Vander Molen, J. Janecke and F.D. Becchetti, 2nd Intern. Conf. on Clustering phenomena in nuclei (College Park, MD, 1975) p. 413;

J.D. Cossairt, R.D. Bent, A.S. Broad, F.D. Becchetti and J. Janecke, Nucl. Phys. A261 (1976) 373.

[3] R.R. Betts, H.T. Fortune, J.N. Bishop, M.N.I. Al-Jadir and R. Middleton, Phys. Lett. 62B (1976) 37; N. Stein, J.W. Sunier and C.W. Woods, Phys. Rev, Lett. 38 (1977) 587.

[4] H.W. Fulbright, C.L. Bennett, R.A. Lindgren, R.G. Markham, S.C. McGuire, G.C. Morrison, U. Strohbusch and J. Toke, Nucl. Phys. A284 (1977) 329.

[5] M. Ichimura, A. Arima, E.C. Halbert and T. Terasawa, Nucl. Phys. A204 (1973) 225.

[6] J.P. Draayer, Nucl. Phys. A237 (1975) 157; K.T.Hecht and D. Braunschweig, Nucl. Phys. A244 (1975) 365 . 
[7] N. Anataraman, C.L. Bennett, J.P. Draayer, H.W. Fulbright, H.E. Gove and J. Toke, Phys. Rev. Lett. 35 (1975) 1131.

[8] M. Conze, Ph.D. Dissertation, Technische Hochschule Darmstadt (1976);

M. Conze and P. Manakos, Phys. Lett. 69B (1977) 29.

[9] A. Arima, M. Harvey and K. Shimizu, Phys. Lett. 30B (1969) 517

[10] R.D. Ratna Raju, J.P. Draayer and K.T. Hecht, Nucl. Phys. A202 (1973) 433.
[11] C.L. Bennett, Nucl. Phys. A284 (1977) 301; C.L. Bennett, H.W. Fulbright, J.F.A. van Hienen, W. Chung and B.H. Wildenthal, to be published.

[12] T. Inoue, Y. Akiy ama and K. Shirai, Proc. Inst. Natural Sciences, Nihon Univ. 12 (1977) 15.

[13] D. Strottman, Nucl. Phys. A1 88 (1972) 488.

[14] D. Strottman, private communication.

[15] D. Braunschweig, Comput. Phys. Commun., to be published.

[16] F.L. Milder, J. Janecke and F.D. Becchetti, Nucl. Phys. A276 (1977) 72. 